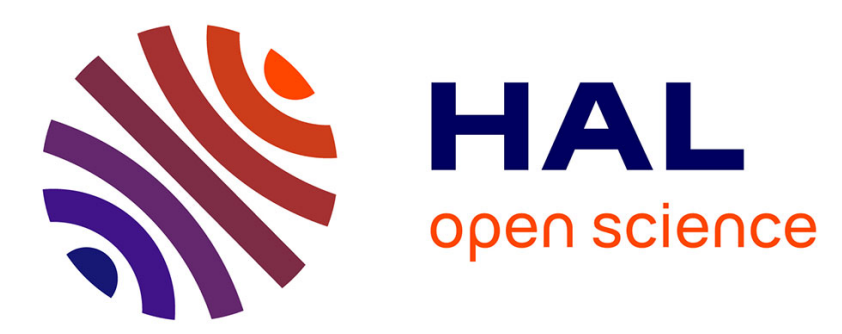

\title{
Effect of locus of control on disordered eating in athletes: The mediational role of self-regulation of eating attitudes
}

Stéphanie Scoffier-Mériaux, Yvan Paquet, Fabienne d'Arripe-Longueville

\section{- To cite this version:}

Stéphanie Scoffier-Mériaux, Yvan Paquet, Fabienne d'Arripe-Longueville. Effect of locus of control on disordered eating in athletes: The mediational role of self-regulation of eating attitudes. Eating Behaviors, 2010, 10.1016/j.eatbeh.2010.02.002 . hal-02524715

\section{HAL Id: hal-02524715 \\ https://hal.univ-cotedazur.fr/hal-02524715}

Submitted on 6 Apr 2020

HAL is a multi-disciplinary open access archive for the deposit and dissemination of scientific research documents, whether they are published or not. The documents may come from teaching and research institutions in France or abroad, or from public or private research centers.
L'archive ouverte pluridisciplinaire HAL, est destinée au dépôt et à la diffusion de documents scientifiques de niveau recherche, publiés ou non, émanant des établissements d'enseignement et de recherche français ou étrangers, des laboratoires publics ou privés. 
6 UFR STAPS - Université de Nice Sophia-Antipolis 261 Route de Grenoble, BP 3259 06205 Nice cedex 03, France

Phone: + 33492296529 

Comportements Alimentaires des Sportifs (LOCSCAS) multidimensionnelle, prenant en compte l'environnement social du sportif. La validité alimentaires et confirme l'importance de la prise en compte des influences sociales.
Résumé
Les travaux en psychologie de la santé considèrent généralement le contrôle comme un facteur protecteur. Ce rôle protecteur a également été montré dans le domaine des comportements alimentaires. Cependant, les précédentes études utilisaient soit l'échelle unidimensionnelle de Rotter (1966), soit l'échelle multidimensionnelle spécifique à la santé (Wallston, Wallston et al., 1978). Aucune échelle spécifique aux comportements alimentaires n'existe. De plus, l'influence de l'environnement social dans les précédentes échelles est limitée. En s'appuyant sur des travaux récents (Paquet et al., 2009 ; Scoffier et al., 2009), l'objectif de cette étude était de proposer une échelle de locus de contrôle convergente et discriminante de cette échelle a été testée en relation avec les comportements

17 Mots clés : Locus de contrôle, Désordres alimentaires, Sport, Validation 
1 Tittle : Validation of a Multidimensional Locus of Control Scale of Eating Attitudes for athletes (LOCSCAS)

Abstract

In the area of health psychology, the control has consistently been considered as a

6 protective factor. This protective role has been also highlighted in eating attitudes' domain.

7 However, current studies use the one-dimensional scale of Rotter (1966) or the

8 Multidimensional Health Locus of Control Scale (Wallston, Wallston, \& De Vellis, 1978),

9 and no specific eating attitudes' scale in the sport context exists. Moreover, the social

10 influence in previous scales is limited. According to recent works (Paquet et al., 2009;

11 Scoffier et al., 2009), the purpose of this study was to propose a Multidimensional Locus of

12 Control Scale of Eating Attitudes for athletes. The scale convergent and discriminate validity

13 was tested in relation with eating attitudes and confirmed the importance of the take in

14 consideration of social influences.

15

16 Keywords: Locus of Control, Eating disorders, Sport, Validation. 
Validation d'une Échelle Multidimensionnelle de Locus de Contrôle Spécifique aux

\section{Introduction}

Le contrôle est un concept majeur en psychologie de la santé (e.g., Bruchon \&

5 Schweitzer, 2002 ; Rascle, Boujut, \& Idier, 2009). Il concerne à la fois l'adoption de

6 comportements sains et l'évitement de conduites à risque, et apparaît comme un facteur

7 protecteur. Le concept de contrôle est aussi relié aux désordres alimentaires (AbuSabha \&

8 Achterberg, 1997 pour une revue). Le terme de désordre alimentaire désigne l'ensemble des

9 attitudes inadaptées liées à l'alimentation, et au contrôle du poids. Ces attitudes s'étendent des

10 restrictions sérieuses, jusqu'aux habitudes de consommation qui aident à perdre du poids ou à

11 maintenir un corps mince (Hobart \& Smucker, 2000). Les désordres alimentaires sont une

12 préoccupation importante pour les adolescents à laquelle les sportifs n'échappent pas (Petrie

$13 \&$ Greenleaf, 2007). Certains sports, pour lesquels la minceur est censée conférer des

14 avantages compétitifs, seraient particulièrement « à risque » (Beals \& Manore, 2000). Tel est

15 le cas, (a) des sports où un faible poids est considéré comme pouvant contribuer à la vitesse

16 et à l'efficience du mouvement (e.g., marathon, courses d'endurance) ; (b) des sports à

17 catégorie de poids; et (c) des sports jugés sur des critères esthétiques qui nécessitent une

18 connaissance de soi poussée et une morphologie spécifique. Rascle et al. (2009) ont montré

19 que maintenir un poids «normalement établi » nécessite des capacités personnelles à

20 contrôler son poids. L'objectif de cette étude était de valider une échelle de locus de contrôle

21 spécifique aux comportements alimentaires chez les sportifs et d'étudier sa relation avec les

22 attitudes alimentaires des sportifs.

23 Le locus de contrôle

24 Le construit de locus de contrôle (LOC) est défini à l'origine par Rotter (1966)

25 comme une expectation de contrôle traduisant le degré de représentation que possède un 
1 individu du lien entre ses comportements et/ou ses caractéristiques personnelles et les

2 renforcements positifs ou négatifs qu'il reçoit. Dans sa conception originale, Rotter (1966)

3 proposait une structure unidimensionnelle distinguant le locus de contrôle interne versus

4 externe. Cette conception a dominé durant plusieurs années. Dans les années 1970, Levenson

5 (1972) a émis des réserves quant à la validité explicative du modèle unidimensionnel de

6 Rotter (1966) et a proposé de considérer trois lieux de contrôle : Interne (i.e., les

7 renforcements obtenus étant liés à nos propres comportements), Personnages tout-puissants

8 (i.e., les renforcements obtenus dépendant du comportement d'autrui significatifs) et

9 finalement la Chance (i.e., les renforcements obtenus dépendant de facteurs externes comme

10 le hasard ou la chance). D'autres auteurs, tels Lecourt (1982) ou plus récemment, Wilkinson

11 (2007) ont confirmé que ce modèle à trois facteurs était plus explicatif et valide que le modèle unidimensionnel original de Rotter (1966). Récemment, plusieurs auteurs (Paquet, 2009 ; Paquet, Berjot, \& Gillet ; 2009) ont proposé un renouvellement conceptuel suggérant

14 la distinction de deux types d'autrui significatifs : d'une part, les « Autres Favorables »

15 susceptibles d'apporter du soutien social à la situation, et d'autre part, les « Autres

16 Défavorables » qui auraient davantage une influence négative par exemple sur la motivation

17 autodéterminée (Paquet et al, 2009). Ces auteurs suggèrent ainsi dans différents domaines

18 (i.e., la performance sportive et le travail) que cette distinction apporterait une meilleure

19 prédiction de l'influence que peut exercer le locus de contrôle.

20 Le Locus de Contrôle et Comportements Alimentaires

21 Le concept de locus de contrôle a été mis en relation avec diverses variables telles

22 l'anxiété (Rossier et al., 2002) ou encore la motivation (Paquet et al., 2009). Le concept de

23 locus de contrôle est aussi un prédicteur des comportements alimentaires (AbuSabha \&

24 Achterberg, 1997 pour une revue). La majorité des travaux entre LOC et comportements

25 alimentaires concernent la relation entre LOC et obésité. Les premières études entre LOC et 
1 obésité remontent aux années 1970, avec Gormanous et Lowe (1975) qui comparent le LOC

2 mesuré, (avec l'échelle de Rotter) de sujets obèses à celui de sujets non-obèses. Ils ne

3 trouvent à l'époque aucune différence ni entres les hommes et les femmes, ni entre les sujets

4 obèses et non-obèses. Quelques années plus tard, Cohen et Alpert (1978) montrent une

5 relation négative entre le score d'externalité à l'échelle de Rotter et la perte de poids.

6 L'externalité serait liée à un risque d'échec du traitement contre l'obésité. Chavez et

7 Michaels (1980) confirment ces résultats avec une échelle spécifique à la santé. En 1982,

8 Saltzer élabore sur le modèle de Rotter une échelle spécifique pour les recherches sur

9 l'obésité. En se servant de cette échelle, Nir et Neumann (1995) retrouvent les résultats

10 antérieurs de Cohen et Alpert (1978) ou Chavez et Michaels (1980), indiquant que

11 l'internalité permettrait de persévérer davantage dans un programme de régime que

12 l'externalité. Le caractère protecteur de l'internalité est une nouvelle fois mis en avant.

13 Concernant le caractère prédicteur du locus de contrôle sur les comportements

14 alimentaires, plusieurs études (e.g., Caggiula \& Watson, 1992 ; Saturnino-Springer \& Bogue,

15 1994) se sont intéressées aux relations entre les comportements alimentaires et les

16 comportements de contrôle du poids et le locus de contrôle relatif au comportement de santé.

17 Les conclusions issues de ces travaux sont assez diverses car les variables utilisées, le

18 contexte ou les sujets diffèrent. Ainsi, il est difficile de généraliser une influence particulière

19 du locus de contrôle sur les comportements alimentaires. Par exemple, Shisslak, Pazda et

20 Crago (1990) montrent que les femmes boulimiques seraient davantage centrées sur un locus

21 de contrôle externe que les femmes non boulimiques du même poids. De la même manière,

22 Griffiths et McCabe (2000) ou, plus récemment, Perry, Silvera, Neilands, Rosenvinge et

23 Hanssen (2008) confirment que le locus de contrôle est un prédicteur important des désordres

24 alimentaires. 
Les différents travaux précédemment cités, bien que parfois inconsistants, révèlent une véritable relation entre le contrôle et les comportements alimentaires. Ces études utilisent en majorité soit l'échelle de Rotter (unidimensionnel et générale), soit l'échelle multidimensionnelle spécifique à la santé (la Multidimensional Health Locus of Control Scale ; Wallston, Wallston, \& De Vellis, 1978), ou encore l'échelle de Saltzer (1982), échelle spécifique à l'obésité et unidimensionnelle. Ces échelles ne sont pas adaptées aux comportements alimentaires à tendance restrictive qui caractérisent les sportifs engagés dans des disciplines «à risque », et suggèrent la nécessité de développer un outil de mesure du Locus de Contrôle Spécifique aux Comportements Alimentaires chez les Sportifs (LOCSCAS).

Afin de proposer un outil de mesure spécifique au contexte sportif, il paraît important de considérer l'environnement social spécifique qui le caractérise et qui est susceptible d'affecter les comportements alimentaires. L'influence de l'environnement social sur les désordres alimentaires a été mis en évidence dans la littérature en psychologie sociale (e.g., Lieberman, Gauvin, Bukowski, \& White, 2001 ; Paxton, Schultz, Wertheim, \& Muir, 1999 ; Shroff \& Thompson, 2006). Ces auteurs montrent une influence significative des pairs, de la famille et des médias sur les désordres alimentaires. Dans la littérature en psychologie du sport, Scoffier, Maiano et d'Arripe Longueville (2009) ont récemment observé une influence distincte de l'entraîneur, des amis et des parents sur les désordres alimentaires. L'entraîneur et les amis auraient une influence positive sur les désordres alimentaires, ils seraient donc vecteurs de patrons mal-adaptatifs. En revanche, les parents seraient négativement reliés aux désordres alimentaires, occupant un rôle protecteur. Considérant ces résultats et les travaux de Paquet et al. (sous 2009), il apparaît que l'entraîneur et les amis pourraient être assimilés aux « Autres Défavorables », alors que la famille et les parents pourraient être assimilés aux « Autres Favorables » du locus de contrôle. 
En résumé, les travaux menés en psychologie sociale montrent une relation

2 significative entre le locus de contrôle et les désordres alimentaires (AbuSabha \& Achterberg,

3 1997). Les travaux de Petrie et Greenleaf (2007) suggèrent que les sportifs seraient une

4 population particulièrement à risque concernant les désordres alimentaires. A ce jour, il

5 n'existe pas d'outil de mesure du locus de contrôle adapté au comportement alimentaire chez

6 les sportifs. Or, l'environnement social spécifique des sportifs et les restrictions sérieuses, ou

7 des habitudes de contrôle du poids dans des sports où la minceur confère des avantages

8 compétitifs, suggèrent la nécessité d'adapter l'échelle multidimensionnelle de mesure du

9 locus de contrôle spécifique à la santé (Wallston, Wallston, \& De Vellis, 1978) au contexte

10 du sport compétitif.

11 Ainsi, l'objet de cette étude était de valider une échelle multidimensionnelle de locus

12 de contrôle à quatre facteurs spécifiques aux comportements alimentaires dans le sport. Cette

13 échelle permettrait de rendre compte de l'influence de deux types de « Personnages tout-

14 puissants »: l'entraîneur et les amis d'un côté, la famille et les parents de l'autre. La validité

15 discriminante et convergente de ces construits (i.e., les quatre facteurs postulés) a été étudiée

16 en relation avec les désordres alimentaires, à partir des hypothèse suivantes : (a) les attitudes

17 alimentaires chez les sportifs devraient être associées négativement au locus de contrôle

18 interne, et positivement au locus de contrôle externe comme le suggèrent les diverses études

19 en psychologie de la santé (Bruchon-Schweitzer, 2002 ;Rascle et al., 2009) ; (b) les « Autres

20 Favorables » (i.e., la famille et les parents ) devraient être reliés négativement aux désordres

21 alimentaires alors que les « Autres Défavorables » (i.e., l'entraîneur et les amis en sport)

22 devraient l'être positivement (Scoffier et al., 2009).

Méthode

24 Participants 

garçons et 71 filles âgés de 18 à 27 ans $\left(M_{\hat{a} g e}=21,12 ; S D=2,87\right)$ pratiquant de manière régulière un sport. Les participants étaient tous français à prédominance caucasienne. Les participants ont tous répondu au questionnaire lors d'une séance de travaux dirigés. La durée de passation n'a pas excédé dix minutes. Ils ont été informés préalablement à la passation du questionnaire du fait qu'ils n'étaient pas obligés de répondre, que l'on conserverait

7 l'anonymat, et que seuls le sexe et la date de naissance étaient rapportés. Il leur a également

8 été signalé qu'il ne s'agissait pas d'un test (i.e., il n'y avait pas de bonnes ou mauvaises

9 réponses), et que les données obtenues ne serviraient qu'à des fins de recherches et

10 demeureraient strictement confidentielles.

11 Mesures été mesuré à l'aide de l'Échelle Multidimensionnelle de Locus de Contrôle Spécifique aux Comportements Alimentaires chez les Sportifs (LOCSCAS) développée pour la présente étude (cf. Tableau 1). Les différents items de l'échelle spécifique aux comportements 16 alimentaires chez les sportifs furent créés par un comité d'experts composé de deux enseignants chercheurs ainsi que l'auteur principal. La nouvelle échelle a été adaptée à partir de la version française de la Multidimensional Health Locus of Control Scale de Wallston et al. (1978) en s'appuyant également sur le modèle à quatre facteurs de l'échelle de Locus de Contrôle spécifique à la Performance en Sport Individuel (Paquet et al., sous 2009). De manière générale, l'adaptation s'est effectuée en remplaçant les mots «maladie (ou être en bonne santé) » par les mots «manger de manière équilibrée ». Deux types d'autrui significatifs furent distingués : l'entraîneur et les amis (autres défavorables), les parents et la

24 famille (autres favorables). L'échelle finale se compose donc de 20 items (cf. Tableau 1), cinq pour chacune des quatre sous-échelles : (a) «Interne », (b) «Entraîneurs, amis », (c) 
1 «Famille, parents » et $(\mathrm{d})$ «Chance ». La dimension « Interne » reflète la relation qu'établit

2 l'individu entre ses comportements et le fait de manger ou non équilibré ; la dimension

3 «Entraîneurs, amis » représente l'influence des comportements des ces derniers sur les

4 comportements alimentaires des individus ; la dimension « Famille, parents » fait référence à

5 l'influence des comportements de ces derniers sur les comportements alimentaires et

6 finalement la dimension «Chance» illustre l'influence des facteurs externes comme la

7 chance ou le hasard sur les comportements alimentaires. La consigne apparaissant sur le

8 questionnaire était: "pour chacune des 20 propositions présentées ci-dessous, indiquez votre

9 degré d'accord, en choisissant entre 4 possibilités : 1: pas du tout d'accord, 2: pas 10 d'accord, 3 : d'accord, 4 : tout à fait d'accord».

11 Attitudes alimentaires. Les attitudes alimentaires ont été mesurées à l'aide de la 12 version française du Eating Attitude Test (EAT) de Garner, Olmsted, Bohr et Garfinkel 13 (1982), validée par Leichner, Steiger, Puentes-Neuman, Perreault et Gottheil (1994), auprès 14 d'une population québécoise francophone. Cet instrument comporte 26 items organisés en 15 trois sous-échelles : (a) restriction alimentaire (e.g., "Je suis terrifié(e) à l'idée d'être trop $16 \operatorname{gros}(\mathrm{se}) »),(\mathrm{b})$ boulimie et obsession de la nourriture (e.g., « Je suis trop soucieux(se) de la 17 nourriture »), et (c) contrôle de la prise alimentaire (e.g., "J'évite de manger quand j'ai 18 faim»). Dans la présente étude, comme c'est le cas dans d'autres travaux antérieurs (e.g., 19 Petrie \& Greenleaf, 2007), un indice global a été considéré, avec une consistance interne 20 satisfaisante (rhô de Jöreskog > ,75).

21 Analyses Tout d'abord, nous avons étudié la validité de construit de l'échelle à l'aide d'une 23 Analyse Factorielle Confirmatoire (AFC). Ensuite, la validité convergente et discriminante a 24 été testée en relation avec les attitudes alimentaires. Relativement au nombre de participants 
1 et afin de maintenir un nombre de degrés de liberté acceptable, il a été décidé de réduire le

2 nombre élevé d'indicateurs par variables manifestes (i.e., les 26 indicateurs de l'échelle EAT

3 était regroupé en trois facteurs) (Bagozzi \& Heatherton, 1994). L'AFC était basée sur 20

4 variables observées et quatre facteurs latents. Les analyses ont été effectuée selon la méthode

5 de maximum de vraisemblance (maximum likelihood) à l'aide du programme LISREL $8.80^{\odot}$

6 (Jöreskog \& Sörbom, 2006). Comme le recommandent plusieurs auteurs (e.g., Byrne, 2005 ;

7 Hu \& Bentler, 1998 ; Vandenberg \& Lance, 2000), les indices d'ajustement suivants ont été

8 utilisés : le chi carré $\left(\chi^{2}\right)$, l'indice comparatif d'adéquation (Comparative Fit Index, $C F I$ ),

9 Non-Normed Fit Index comme indice incrémental (NNFI, Bentler \& Bonette, 1980), les

10 résidus standardisés (Root Mean Square Error of Approximation, RMSEA) et (Root Mean

11 Square Residual, RMR). Les valeurs $\geq, 90$ et $\geq$,95, pour le CFI et le NNFI, sont respectivement considérées comme des indices d'ajustement très satisfaisants du modèle testé (e.g., Byrne, 2005 ; Hu \& Bentler, 1998 ; Vandenberg \& Lance, 2000). Concernant le RMSEA et le RMR, les valeurs comprises entre ,05 et ,08 sont considérées comme des indices d'ajustement satisfaisants du modèle testé (e.g., Byrne, 2005 ; Vanderberg \& Lance, 2000). Enfin, la fiabilité a été éprouvée avec le rhô de Jöreskog (1971).

Statistiques Descriptives

Dans un premier temps, des statistiques descriptives (i.e., moyenne, écart-type, score minimum et maximum) ont été réalisées pour chaque item de la LOCSCAS. Les résultats montrent que l'ensemble des scores possibles pour chaque item a été utilisé. Même si certaines moyennes semblent élevées ou faibles, elles reflètent une tendance à observer des scores élevés pour la dimension «Interne » et des scores plus faibles pour la dimension

24 «Chance ». Ainsi, on observe des moyennes aux scores de chaque item allant de 1,54 à 3,70 sur une possibilité de 4,00 et des écarts-types allant de ,74 à 1,12. 
1 Analyse Factorielle Confirmatoire de l'échelle Locus de Contrôle Spécifique aux

\section{Comportements Alimentaires chez les Sportifs}

Une AFC a été réalisée à l'aide du logiciel LISREL $8.80^{\odot}$ afin de tester la validité de la structure factorielle de l’Échelle Multidimensionnelle de Locus de Contrôle Spécifique aux Comportements Alimentaires chez les Sportifs (LOCSCA). Les différents indices d'ajustement $\left(\chi^{2}(179,158)=237,75, \mathrm{CFI}=, 91, \mathrm{NNFI}=, 90, \mathrm{RMR}=, 07, \mathrm{RMSEA}=, 05\right.$, Intervalle de Confiance (IC) pour le RMSEA = ,039/,067) sont conformes aux normes usuelles (Kline, 1998 ; Roussel, Durrieu, Campoy, \& El Akremi, 2002). La consistance interne de chaque facteur a été vérifiée à l'aide des Rhô de Jöreskog qui ont été calculés. Les valeurs pour l'ensemble des facteurs étaient satisfaisantes : (a) ,66 pour le locus Interne; (b) ,75 pour la Famille et les Parents ; (c) ,77 pour l'Entraîneur et amis et (d),66 pour la Chance. Validité Discriminante et Convergente des Construits Analyse de la validité de construit du modèle testé. L'AFC met en évidence que le modèle hypothétique testé est acceptable $\left(\chi^{2}(179,214)=354,58 ; \mathrm{CFI}=, 87 ; \mathrm{NNFI}=, 85\right.$; $\mathrm{RMR}=, 07 ; \mathrm{RMSEA}=, 06$; Intervalle de Confiance $(\mathrm{IC})$ pour le RMSEA = ,052/,074). Tous les $\lambda$ s sont significatifs $(t>1,96)$

Modélisation d'équations structurales. Afin de tester la validité discriminante et convergente des construits, un modèle d'équations structurales a été réalisé à l'aide du logiciel LISREL $8.80^{\odot}$ permettant de tester l'influence du locus de contrôle sur les attitudes alimentaires. Le modèle (voir figure 1) présente des indices d'adéquation satisfaisant $\left(\chi^{2}\right.$ $(179,209)=323,12, \mathrm{CFI}=, 91, \mathrm{NNFI}=, 90, \mathrm{RMR}=, 07, \mathrm{RMSEA}=, 06$, Intervalle de Confiance (IC) pour le RMSEA = ,043/,067). 
Le modèle permet de montrer, d'une part, l'influence positive qu'exercent l'entraîneur

et les amis sur les attitudes alimentaires, confirmant ainsi le rôle défavorable que peuvent avoir l'entraîneur ou les amis dans l'apparition de désordres alimentaires chez les sportifs. D'autre part, le rôle plutôt favorable et protecteur de la famille et des parents est également mis en avant. Ces deux résultats confirment ceux déjà trouvés par Scoffier et al. (2009). Les dimensions Interne et Chance ne sont pas reliées aux attitudes alimentaires.

\section{Discussion}

L'objet de cette étude était d'adapter une échelle multidimensionnelle de locus de contrôle spécifique aux comportements alimentaires des sportifs. Actuellement, les échelles les plus utilisées dans l'étude des comportements alimentaires sont celles de Rotter (1966) ou encore la Multidimensional Health Locus of Control Scale (MHLCS ; Wallston et al., 1978).

Ces échelles ne prennent pas en comptent la spécificité de l'environnement social, en particulier dans le domaine sportif. Sur la base de la nouvelles conceptualisation du locus de contrôle de Paquet et al. (2009), et des travaux de Scoffier et al. (2009), nous avons donc établi une échelle de LOC multidimensionnelle à quatre facteurs, dans laquelle l'entraîneur et les amis seraient considérés comme des «Autres Défavorables », tandis que les parents et la famille seraient considérés comme des «Autres Favorables ». Ainsi, la nouvelle échelle a été adaptée à partir de la MHLCS (Wallston et al., 1978) sur un modèle à quatre facteurs : Interne, Autres Défavorables Autres Favorables et Chance. Une analyse factorielle confirmatoire a été réalisée afin de tester l'adéquation de nos données et du modèle théorique à quatre facteurs. Le modèle présente des indices d'adéquation corrects et conformes aux normes habituellement utilisées (Kline, 1998 ;

24 Roussel et al., 2002). La consistance interne des différents facteurs de notre échelle a été 
1 vérifiée à l'aide des rhô de Jöreskog (1971). La structure factorielle de cette adaptation de la

2 MHLCS est donc vérifiée.

Ainsi, afin de tester la validité des construits constituant l'échelle proposée (LOCSCAS), les relations entre les quatre facteurs de notre échelle de locus de contrôle et les comportements alimentaires ont été étudiées. Une analyse en équation structurale et permet

6 de confirmer les résultats de Scoffier et al. (2009). En effet, les résultats confirment

7 l'influence « défavorable » de l'entraîneur et des amis sur les comportements alimentaires et

8 le rôle protecteur des parents et de la famille. Ces résultats attestent bien la validité

9 discriminante et convergente de l'échelle de locus de contrôle à quatre facteurs spécifique

10 aux comportements alimentaires des sportives proposée par dans cette étude. De plus, cela

11 confirme l'importance de la distinction entre les « Autres Favorables » et les « Autres

12 Défavorables » dans les études portant sur le locus de contrôle. L'absence de lien significatif

13 entre les dimensions Interne et Chance et les comportements alimentaires soulèvent

14 différentes questions : (a) les facteurs sociaux prennent peut-être une place plus importante

15 que les facteurs individuels ? (b) l'influence des dimensions Interne et Chance sur les

16 comportements alimentaires est peut-être modérer par d'autres variables comme par exemple

17 l'autorégulation?

En résumé, dans le domaine de la santé, comme dans celui des comportements

19 alimentaires, les relations avec le locus de contrôle ont souvent été abordées sur le versant de

20 l'internalité. Or l'importance de l'environnement social est loin d'être négligeable. Ainsi, 21 comme suggéré par Levenson (1972), l'ajout de la dimension d'autrui significatifs est très important. Les travaux récents de Paquet et collaborateurs (2009) montrent l'importance de la

23 distinction entre les autrui significatifs ayant une influence favorable et ceux ayant une

24 influence plus négative. La présente étude confirme l'importance de cette distinction. De

25 plus, cette distinction permet de montrer l'importance que peuvent jouer les parents ou la 
1 famille, probablement en terme de soutien social, face aux problèmes alimentaires rencontrés

2 par les sportifs. Néanmoins, le rôle majeur reste celui des entraîneurs et des amis dont

3 l'influence négative mérite encore d'être interrogée.

Plusieurs limites doivent être prises en considération en interprétant ces résultats.

5 D'abord, les données sont des données auto-rapportées et ont pu avoir été influencées par la

6 désirabilité sociale. Puis, la seconde partie de l'étude était réalisée selon une procédure cross

7 sectionnal qui limite la stabilité dans le temps des rapports entre les variables. De plus,

8 davantage de recherches sont nécessaires pour confirmer la validité de notre échelle sur

9 d'autres athlètes et pour déterminer son niveau d'adaptation. D'abord, la validité du LOCSCA

10 devrait être testée auprès d'adolescents et, au besoin, un instrument approprié selon l'âge des

11 sujets devrait être développé. Il serait également intéressant de valider cet instrument en anglais pour permettre des études transculturelles. En second lieu, la validité externe devrait être examinée en associant le locus de contrôle relatifs aux comportements alimentaires des sportifs avec d'autres variables pertinentes, telles l'autorégulation des comportements alimentaires ou les styles attributionnels.

Pour conclure, l'échelle multidimensionnelle de locus de contrôle spécifique aux comportements alimentaires des sportifs paraît être un outil intéressant permettant d'appréhender les processus de contrôle des comportements alimentaires. Cette échelle spécifique possède l'avantage de tenir compte de l'environnement social du sportif (i.e., entraîneur, amis et famille) permettant une meilleure prédiction de l'influence qu'exerce le

21 locus de contrôle sur les comportements alimentaires. Ces résultats suggèrent la mise en place de formations destinées aux entraîneurs sur les comportements à tenir face à leurs sportifs. De plus, une éducation au contrôle de leurs comportements alimentaires pourrait être développée auprès des sportifs à risque (voir par exemple, Rascle et al., 2009). 


\section{Références}

2 AbuSabha, R., \& Achterberg, C. (1997). Review of self-efficacy and locus of control for nutrition and health-related behavior. Journal of American Dietetic Association, 97, $1122-1132$.

Bagozzi, R. P., \& Heatherton, T. F. (1994). "A general approach to representing multifaceted personality constructs: Application to self esteem," Structural Equation Modeling, 1, $35-67$.

Beals K. A., \& Manore, M. M. (2000). Behavioral, psychological and physical characteristics of female athletes with subclinical eating disorders. International Journal of Sport Nutrition \& Exercise Metabolism, 10, 128-143.

Bentler, P. M., \& Bonett, D. G. (1980). Significant tests and goodness of fit in the analysis of covariance structures. Psychological Bulletin, 88, 588-606.

Bruchon-Schweitzer, M. (2002). Psychologie de la santé : modèles, concepts et méthodes. Paris: Dunod.

Byrne, B. (2005). Factor analytic models: Viewing the structure of an assessment instrument from three perspectives. Journal of Personality Assessment, 85, 17-32.

Caggiula, A. W., \& Watson, J. E. (1992). Characteristics associated with compliance to cholesterol lowering eating patterns. Patient Education and Counseling, 19, 33-41.

Chavez, E. L., \& Michaels, C. (1980). Evaluation of the health locus of control for obesity treatment. Psychological Reports, 47, 709-710.

21 Cohen, N. L., \& Alpert, M. (1978). Locus of control as a predictor of outcome in treatment of obesity. Psychological Reports, 42, 805-806.

Garner, D., Olmsted, M., Bohr, Y., \& Garfinkel, P. (1982). The Eating Attitude Test: psychometric features and clinical correlates. Psychological Medicine, 12, 871-878. 
1 Gormanous, G. K., \& Lowe, W. C. (1975). Locus of control and obesity. Psychological Reports, 37, 30.

3 Griffiths, J. A., \& McCabe, M. P. (2000). The influence of significant others on disordered eating and body dissatisfaction among early adolescent girls. European Eating Disorders Review, 8, 301-314.

6 Hobart, J. A., \& Smucker, D. R. (2000). The female athlete triad. American Family Physician, 61, 3357-3364.

Hu, L., \& Bentler, P. M. (1998). Fit indices in covariance structure modelling: Sensitivity to underparametrized model misspecification, Psychological Methods, 3, 424-453.

Jöreskog, K. G. \& Sörbom, D. (2006). LISREL 8.80 for Windows [Computer Software]. Lincolnwood, IL: Scientific Software International, Inc.

Jöreskog, K. G. (1971). Statistical analysis of sets of congeneric tests, Psychometrika, 36, $109-133$.

Kline, R. B. (1998). Principles and practices of structural equation modeling. New York, NY: Guilford Press.

Lefcourt, H. M. (1982). Locus of control: Current trends in theory and research (2e ed.). Hillsdale: Lawrence Erlbaum.

Leichner, P., Steiger, H., Puentes-Neuman, G., Perreault, M., \& Gottheil N. (1994). Validation d'une échelle d'attitudes alimentaires auprès d'une population québécoise francophone. Revue Canadienne de Psychiatrie, 39, 49-54.

Levenson, H. (1972). Distinctions within the concept of internal-external control: development of a new scale. Proceedings of the 80th annual convention of the American Psychological Association, 261-262. 
1 Lieberman, M., Gauvin, L., Bukowski, W. M., \& White, D. R. (2001). Interpersonal influence and disordered eating behaviors in adolescent girls: The role of peer modeling, social reinforcement, and body-related teasing. Eating Behaviors, 2, 215-236.

Nir, Z., \& Neumann, L. (1995). Relationship among self-esteem, internal,external locus of control, and weight change after participation in a weight reduction program. Journal of Clinical Psychology, 51, 482-490.

Paquet, Y. (2009). Les différents construits de la notion de contrôle in Y. Paquet (Eds), Psychologie du contrôle : Aspects théoriques et applications. Bruxelles : DeBoeck.

Paquet, Y., Berjot S., \& Gillet, N. (2009). Validation d'une échelle de locus de contrôle spécifique à la performance en sport individuel (LOCPSI), Bulletin de psychologie, 62(4), 351-363.

Paxton, S.J., Schutz, H.K., Wertheim, E.H., \& Muir, S.L. (1999). Friendship clique and peer influences on body image concerns, dietary restraint, extreme weight-loss behaviours, and binge-eating in adolescents girls. Journal of Abnormal Psychology, 108, 255-266.

Perry, J. A., Silvera, D. H, Neilands, T. B, Rosenvinge, J. H., \& Hanssen, T. (2008). A study of the relationship between parental bonding, self-concept and eating disturbances in Norwegian and American college populations. Eating Behavior, 9, 13-24.

Petrie, T. A., \& Greenleaf, C. A. (2007). Eating disorders in sport: From theory to research to intervention. In G. Tenenbaum (Ed.), Handbook of Sport Psychology, 3rd Edition (pp. 352-378). J. Wiley \& Sons, Inc, Hoboken, New Jersey.

Rascle, N., Boujut, E. \& Idier, L. (2009). Contrôle et santé. In Y. Paquet (Eds), Psychologie du contrôle : Aspects théoriques et applications. Bruxelles : DeBoeck.

Rossier, J., Rigozzi, C., \& Berthoud, S. (2002). Validation de la version française de l'échelle de contrôle de Levenson (IPC), influence de variables démographiques et de la personnalité. Annales Medico Psychologiques, 160, 138-148. 
1 Rotter, J. B. (1966). Generalized expectancies for internal versus external control of reinforcement. Psychological Monograph, 80, 1-28.

3 Roussel, P., Durrieu, F., Campoy, E., \& El Akremi, A. (2002). Méthodes d'Equations

Saltzer, E. B. (1982). The Weight Locus of Control (WLOC) scale: A specific measure for obesity research. Journal of Personality Assessment, 46, 620-628.

Saturnio-Springer, N., \& Bogue, N. (1994). Nutrition locus of control and dietary behavior of pregnant women. Applied Nursing Research, 7, 28-31.

Scoffier, S., Maïano, C., \& Arripe-Longueville, F. (d') (2009). The effects of social Disorders, DOI: 10.1002/eat.20597.

Shisslak, C. M., Pazda, S. L., \& Crago, M. (1990). Body weight and bulimia as discriminators of psychological characteristics among anorexic, bulimic, and obese women. Journal of Abnormal Psychology, 99, 380-384.

Shroff, H., \& Thompson, J. K. (2006). The tripartite influence model of body image and eating disturbance: A replication with adolescent girls. Body Image: An International Journal of Research, 3, 17-23.

Vandenberg, R. J. \& Lance, C. E. (2000). A review and synthesis of the measurement invariance literature: Suggestions, practices, and recommendations for organizational research. Organizational Research Methods, 3, 4-69.

Wallston, K. A., Wallston, B. S., \& DeVellis, R. (1978). Development of the multidimensional health locus of control scales (MHLCS). Health Education Monographs, 6, 160-170. 
1 Wilkinson, W. W. (2007). The structure of the Levenson locus of control scale in young 2 adults: Comparing item and parcel indicator models. Personality and Individual Differences, 43, 1416-1425.

4 
1 Table 1. Descriptive Statistics, Coefficients of Internal Consistency (Cronbach alpha) and

2 Inter-Scale Correlations.

\begin{tabular}{lccccccccccc}
\hline & 1 & 2 & 3 & 4 & 5 & 6 & 7 & 8 & 9 & 10 \\
\hline $\mathrm{M}$ & 3.288 & 1.84 & 2.31 & 1.88 & 3.956 & 3.652 & 3.885 & 3.413 & 3.269 & 2.291 \\
$\mathrm{SD}$ & .484 & .660 & .698 & .598 & 1.204 & 1.308 & 1.384 & 1.747 & 1.919 & .861 \\
$\mathrm{M}_{\text {females }}$ & 3.379 & 1.772 & 2.338 & 1.621 & 3.81 & 3.84 & 4.05 & 3.00 & 4.52 & 2.415 \\
$\mathrm{SD}_{\text {females }}$ & .363 & .722 & .750 & .425 & 1.258 & 1.393 & .759 & 1.32 & 1.47 & .856 \\
$\mathrm{M}_{\text {males }}$ & 3.215 & 1.884 & 2.291 & 2.050 & 3.873 & 4.049 & 4.064 & 2.987 & 4.645 & 2.217 \\
$\mathrm{SD}_{\text {males }}$ & .536 & .577 & .624 & .588 & 1.455 & 1.437 & .684 & 1.411 & 1.365 & .805 \\
$\alpha$ & .66 & .77 & .74 & .66 & .76 & .79 & .78 & .77 & .74 & .76 \\
\hline
\end{tabular}

1. Internal locus

2. External locus: Coach \&

Friends

3. External locus: Family

$-046$

4. External locus: Luck

$.003 .381 * * \quad-$

5. Self-regulation in context of

food temptation

6. Self-regulation in context of negative affect

$-.189 * \quad .177 *-.032 \quad-$

$.080-.184 *-.185 *-.158 * \quad-$

$\begin{array}{llllll}.078 & -.124 & -.009 & -.064 & .366^{* *} & -\end{array}$

7. Self-regulation in context of social interaction

8. Self-regulation in context of compensatory strategies

$\begin{array}{llllll}.227 * * & -.186 * & .084 & -.040 & .228 * * & .279 * *\end{array}$

$\begin{array}{lllllll}-088 & -.014 & .023 & .003 & .014 & -.012 & .002\end{array}$

9. Self-regulation in context of lack of anticipation of consequences on performance

$\begin{array}{llllllll}.082 & -.341 * * & -.039 & .033 & -.079 & .245^{* *} & .348 * * & .036\end{array}$

10. Eating disorders

$\begin{array}{lllllllll}-.084 & .334 * * & -.049 & .114 & .038 & -.285 * * & -.190 * & .113 & -.596 * *\end{array}$

Notes. M: means; SD: standard deviation; $\alpha$ : Cronbach alpha; $* p<.05 ; * * p<.01$. 